\title{
Vascular Complications in Live Related Renal Transplant - BSMMU Experience
}

\author{
Tohid Mohammad Saiful Hossain ${ }^{1}$, Tahmina Karim², Md. Habibur Rahman ${ }^{3}$, AKM Khurshidul Alam ${ }^{4}$, \\ Abdul Matin Anamur Rashid Choudhury ${ }^{5}$
}

Received: 20 - 07 - 2020

Accepted: 11 - 09 - 2020

Conflicts of interest: None

Keywords: Live related Renal transplantation, Vascular complication

\begin{abstract}
Introduction: Renal Transplantation is the optimal treatment for end-stage renal disease. Among the surgical complication in Renal Transplant vascular complication is most dreaded and many lead to sudden allograft loss and increase patient morbidity and even mortality.

Objective: To share our experience and analysis of outcome of vascular complications of Live related Renal Transplant in our Bangabandhu Sheikh Mujib Medical University (BSMMU), Dhaka.

Materials \& Methods: One hundred eighty two (182) live related Renal Transplant were performed from July 2011 to March 2020. During this period we evaluated this cases retrospectively to find out vascular complications and analyzed them for frequency, clinical presentation and their management and outcome.

Result: The age of recipients range from 6-65 years, male 129, female 53 vascular complications are found in 8 cases - 1 Renal Artery Thrombosis, 2 venous thrombosis, 1 Renal Artery aneurysm, 1 hematocele, 3 lymphocele. 4 patients needed graft nephrectomy, 2 lymphocele cases need lympho-peritoneal shunt and 1 improve by sclerotherapy with povidone-iodine, hematoma needs surgical evacuation. One patient died after graft Nephrectomy due to sepsis.

Conclusion: Major vascular complications are relatively uncommon after live related Renal Transplantation but still contributes a major factor of graft loss and patient morbidity. According to these data we can conclude Renal Transplantation is safe procedure for end stage renal disease.
\end{abstract}

\section{Introduction:}

After so many experimental studies the first ${ }^{1,2}$ human successful kidney transplantation performed by Joseph Murray in 1954 between identical twins. ${ }^{3}$ The evaluation of surgical Anastomotic techniques and immunosuppressive regimes have established renal transplantation is a safe procedure for end stage kidney diseases.
Complications after Renal Transplantation can be divided into pathological and surgical. Among surgical - vascular complications are most dramatic and dreaded and leads to sudden graft loss $^{4}$ in early postoperative period, increases morbidity and even mortality. Reported vascular complications include renal artery thrombosis \& stenosis, Renal vein thrombosis, aneurysms, hematomas, lymphocele and arterio venous fistula. ${ }^{5}$

1. Associate Professor of Urology \& Renal Transplantation Surgery, Bangabandhu Sheikh Mujib Medical University, Dhaka.

2. Associate Professor of Paediatric Cardiology, Bangabandhu Sheikh Mujib Medical University, Dhaka.

3. Professor of Urology \& Renal Transplantation Surgery, Bangabandhu Sheikh Mujib Medical University, Dhaka.

4. Professor \& Chairman of Urology, Bangabandhu Sheikh Mujib Medical University, Dhaka.

5. Associate Professor, Department of Urology, National Institute of Kidney Disease \& Urology, Sher-e-Bangla Nagar, Dhaka Correspondence: Dr. Tohid Mohammadd Saiful Hossain, Associate Professor of Urology and Renal Transplantation Surgery, Bangabandhu Sheikh Mujib Medical University, Dhaka. Email: dr.tmsh @yahoo.com, dr.tmsh@gmail.com 
In this study we want to share our experience of such complications in 182 live related renal transplantation and analyze the outcome with the hope to increase awareness of transplant surgical team.

\section{Material and Methods:}

One hundred and eighty two live related renal transplantation were done at our institute from July 2011 to March 2020 overcoming so many hurdles and ups and downs of live related renal transplantation program in our Country. Among them 10 were Paediatric transplant. 16 cases were multiple arteries (9 double \& 7 triple). All transplants done in right iliac fossa except Paediatric those a bit higher. In cases of adult single artery and vein anastomosed to external illiac artery and vein, by using $6 / 0$ and $5 / 0$ prolene in end to side fashion. In Paediatric cases 6 were anastomosed to abdominal Aorta and inferior vena cava and 4 to common illiac vessels. Multiple vessels were dealt with either two/three separate anastomosis or as two joined into single pantaloon anastomosis depending upon the anatomical findings. In all cases ureteroneocystostomy was performed by modified Lich Gregor Technique over JJ stent. Immunosuppressive regimes were given according to standard international protocols usually 3 drug regimen, prednisolone, mycophenolate mofetil, cyclosporine/ tacrolimus and in special cases Baxiliximub as induction therapy.

Postoperatively patients were kept in a dedicated transplant intensive care unit till discharge. Complete blood count, renal function tests and other serum biochemical parameters, serum drug level done routinely and urgently depending on clinical ground. Ultrasound and color doppler was performed when there is any suspicion of impaired or abnormal transplant kidney function. Central venous line Catheters, drains, per urethral catheter and JJ stent removed in time according to our institutional protocol.

In the present study, a retrospective analysis of inpatient and follow up records of vascular complications of all patients was evaluated and cases in which early graft loss due to acute rejection and acute tubular necrosis (ATN) were excluded from analysis of vascular complication. The incidence, time of onset and clinical presentation, diagnosis, management and ultimate outcome of the patient and graft in all cases were studied.

\section{Results:}

In our series out of 182 transplant recipients of live related kidney with age ranging 6-65 years, among them male 129 and female 53 vascular complications occurred in $8(4.39 \%)$, complications are divided in two categories (type 1 \& type 2) depending on graft loss. Table 1 summarizes the rates of vascular complications following renal transplant in our centre.

Overall we noticed 4 major vascular complications leading to allograft loss (type-1). Among them 1 renal artery thrombosis, 2 renal vein thrombosis and 1 renal artery aneurysm onset of presentation of thrombosis had most dramatic and dread. All cases of renal vein thrombosis occurred within first 5 days and renal artery thrombosis within first week. Renal artery aneurysm formation were diagnosed in $3^{\text {rd }}$ week after transplantation. Renal artery thrombosis had sudden anuria with minimal or no abnormal discomfort. On the other hand venous thrombosis presented as oliguria with severe graft pain and tenderness. Renal artery aneurysm has similar presentation with failure of serum creatinine to return to baseline. All 4 cases are diagnosed by color doppler ultrasound. Immediate surgical exploration was done in all cases after diagnosis but unfortunately all the cases need graft nephrectomy. In all cases of thrombosis histopathology revealed cortical necrosis, unfortunately one patient died within 7 days after graft nephrectomy.

In type- 2 complications- 1 developed hematoma in $2^{\text {nd }}$ weeks after transplant presenting with anemia oliguria and peri-renal huge echogenic collection with hydronephrosis of transplant kidney. Ultrasonography guided aspiration tried but failed after that surgical explanation done and hematoma evacuated.

Lymphocele developed after 6 weeks with peri-renal collection with heaviness in right illiac fossa and swelling at inguinal region, oedema of ipsilateral leg with oliguria and hydronephrosis of transplant kidney. 1 case improved by ultrasono guided aspiration and sclerotherapy by povidone iodine and other 2 need open lympho-peritoneal shunt.

Table-I : Vascular complications following Renal Transplant

\begin{tabular}{llll}
\hline Type-1 (Rapid graft loss) & & Type-2 (graft survives after treatment) & \\
\hline Renal artery thrombosis & 1 & Hematoma & 1 \\
Renal vein thrombosis & 2 & Lymphocele & 3 \\
Renal artery aneurysm & 1 & & \\
\hline
\end{tabular}




\section{Discussion:}

Though vascular complications is uncommon after renal transplantation but it is grievous in relation to outcome. The cause of these complication is multifactorial. Some may be due to technical factors, others are recipient related such as hypercoagulopathics and decreased cardiac output.

Reported incidence of transplant renal artery thrombosis is $.5-3.5 \% .{ }^{6}$ Sudden caesation of urine production is usual presentation without any other signs or symptoms in early postoperative period. Immediate ultrasound of transplanted kidney and color doppler study reveals complete absences of arterial blood flow ${ }^{7}$ and thus exclude other common cause like acute rejection or ATN. Transplant renal artery thrombosis is usually due to technical factorsresulting from twisting or kinking of anastomosis, injury to intima, may be during donor artery perfusion, poor cardiac output, thrombotic events, acute rejection episodes, external compression by lymphocele, hematomas, toxicity of immunosuppressive agents such as cyclosporin or sirolimus. ${ }^{8,9}$ Transplant renal artery thrombosis is a surgical emergency needs urgent exploration \& with restoration of blood flow to the kidney. In most of the cases diagnosis is too late leads to graft nephrectomy. In our series we diagnose 1 renal artery thrombosis needs graft nephrectomy. We recognize technical factors was the cause which correlates with others findings. ${ }^{10}$

Renal vein thrombosis is a grave early complication of kidney transplantation leading to graft loss in almost all cases ${ }^{11}$ with reported incidence of .5 to $6 \% .^{12}$ Sudden oliguria, hematuria associated with pain in graft site and tenderness over graft with graft swelling even rupture of kidney can result in severe hemorrhage is the usual presentation of renal vein thrombosis. Several factors have been identified as an causative agent like - right donor kidney with short thin walled renal vein use of left illiac fossa, hypercoaguable states such as deficiency of antithrombosis III, protein C, protein S, relatively young or old donor age, diabetes mellitus, peritoneal dialysis, long ischemic time, ATN[11]. Diagnosis is confirmed by color doppler which shows a complete absence of venous flow and abnormal aterial signal with a plateaulike reversed diastolic flow as well as enlarged renal graft. ${ }^{13} \mathrm{Immediate}$ exploration is required to venous thrombectomy and restore blood flow if not possible immediate graft nephrectomy to save the life. In our study we encountered 2 cases of renal vein thrombosis which is much less than the reported incidence of most of the series ${ }^{10}$ may be due to only live related transplant. We manage those by graft nephrectomy unfortunately 1 patient died due to sepsis $7^{\text {th }}$ postoperative day. We identified that right donor kidney with short thin walled renal vein was the causative factor for renal vein thrombosis.

Transplant renal artery aneurysm is a rare complication with reported incidence less than $1 \% .{ }^{14}$ It usually results from anastomotic leakage due to defective suture technique or damage to artery wall by trauma, vessel wall ischemia or infection. ${ }^{15}$ Clinically the aneurysm is usually asymptomatic and found incidentally on color doppler study. They may present with fever, anemia or graft dysfunction due to mass effect. It is potentially a fatal condition leading to sudden rupture to torrential haemorrhage. Therapeutic options are - careful follow-up, endovascular treatment, open surgical repair/ graft Nephrectomy. In our series we encountered 1 cases which was managed by graft nephrectomy. Mycotic aneurysm was histopathologically proven which is similar with other findings. ${ }^{10}$

Hematomas are minor complications occurring in the early postoperative period. ${ }^{5}$ It usually occurs due to small leakage at the anastomosis sites or minor bleeding from the surface of renal graft or from surrounding tissues, often they present late due to graft needle biopsy. Usually hematomas are small and asymptomatic but when becomes enlarged and organized may give with mass effect leading to graft dysfunction. Diagnosis confirmed by ultrasound examination which reveals highly echogenic mass. In our study we encountered 1 case of hematoma which was mentioned earlier and managed by exploration and evacuation and on exploration no active bleeding was found.

Lymphocele formation in post kidney transplantation is a common and well known complication with reported incidence $1-26 \% .{ }^{16}$ It is usually caused by accumulation of lymphatic fluid due to transection of lymphatic vessels during recipient bed preparation, during donor nephrectomy. In our series we diagnosed 3 cases of lymphocele around 6 weeks their mode of presentation was mentioned earlier and diagnosed by ultrasound and chemical analysis of aspirated fluid excludes urine. We managed 2 cases by open lymphoperitoneal shunt and other by ultrasound guided aspiration and Sclerotherapy by povidone iodine. We thought technical fault in surgical technique is the 
factor for excessive lymphatic fluid accumulation leading to lymphocele formation.

In our series we did not find any renal artery stenosis. It is probably due to lack of long term follow-up and or not doing routine color doppler study in follow-up.

\section{Conclusion:}

Major vascular complication is rare after live related kidney transplantation but still it is the important cause of early postoperative graft loss. Per-operative doppler study \& prompt evaluation and emergency color doppler study and selective renal transplant angiography are required for graft preservation. More over meticulous surgical technique during donor and recipient surgery including bench and perfusion, retrieval of right donor renal vein with cuff of inferior venacava, advances in interventional radiology \& cardiology have significantly reduced these complications to make the renal transplantation a safe procedure for end stage renal disease.

\section{References:}

1. Carrel A: La technique des anastomosis vascularizes et la transplantation des visceres. Lyon Med 98:859. 1902.

2. Morre PD: Give and Take: The development of tissue transplantation. Philadelphia: W.B. Saunders; 1964

3. MerriII, JP, Murray JE, Harrijon JH: Sucessful homo transplntation of human kidney between identical twins, JAMA 160:277, 1956.

4. Humar A, Key N, Ramcharan I, payne WD, Suthetiand DE, Mathas AJ: Kidney transplantation after initial graft loss to vascular thrombosis clin transplant: 2001, 15:16-10.

5. D. Dimitroulis, J. Bokos, G. Zavos, N. Nikiteas, N.P Karidis, P. Katsaronis and A. Kostakis: vascular complications in renal transplantation: A single centre experience in 1367 renal transplantations and review of the literature Transplant proceedings, 41, 1609-1614(2009).

6. Rouviere O, Berger P, Bezian C, Gernier JL et al. Acute thrombosis renal artery: Graft salvage by means of intra-arterial fibrinolysis. Transplantation 73:403, 2002.

7. Baxter GM: ultrasound of renal Transplantation Clin RadioL 56:802, 2001.

8. Groggel OG: Acute Thrombosis of the renal transplant artery: a case report and review of the literature. Clin NephroL 36:42, 1991.

9. Beyga ZT, Kahan BD: Surgical complications of renal transplantation. J NephroL 11:137, 1998.

10. Aneesh Srivastava, Jantinder Kumar, Sandeep Sharma, Abishek, MS Ansari, Rakesh Kapoor, Vascular Complication in live related renal transplant: An experience of 1945 cases. 29(1), 4247, 2013.

11. P. Giustacchini, F. Pisanti, F. Citterio A.M, De Gaetaso, M. Castagneto and G. Nassi - Renal vein Thrombosis after renal transplantation: An important cause of graft loss. Transplant proceedings, 34, 2126-2127(2002).

12. Robertson AJ, Nargund V, Gray DW, Morris PJ: Low dose aspirin as prophylaxis against renal vein thrombosis in renal transplant recipients. NephroL Dia Transplant: 2000:15:1885-6.

13. Kaveggia LP, Parrella RR, Grant EG, et al: Duplex Doppler sonography in renal allografts: the significance of reversed flow in diastolic. Am J Roendgeno L 155:295, 1990.

14. Koo CK, Rodger S, Baxter GM, Extrarenal pseudoaneurysm: An uncommon complication following renal transplantation. Clin RadioL 1999:54, 755-8.

15. Fujkata S, Tanji N, Iseda T, Onoka H, Yoko Yama M: Mycotic aneurysm of the renal transplant artery. Int J Urol 2006:13:820-3.

16. Hany EI-Hennawy, Christian C, Morrill, Giuseppe Orlando and Alan C, Farncy: Vascular complications in renal transplantation kidney transplantation, Bioengineering and regeneration. https://dx.dio.org/10.1016/B978-0-12801734'"0.00034-5. 